\title{
Construcción y Calibración de una Celda para la Determinación de la Distribución de Tamaño de Micro Partículas
}

\author{
Gonzalo E. Salinas-Salas y Fernando F. Espinosa \\ Universidad de Talca, Facultad de Ingeniería, Camino Los Niches km. 1, Curicó-Chile. \\ (e-mail: gsalinas@utalca.cl; fespinos@utalca.cl)
}

Recibido Sep. 29, 2010; Aceptado Nov. 23, 2010; Versión Final recibida Dic. 15, 2010

\section{Resumen}

Se muestra y analiza el desarrollo y los resultados alcanzados al construir y calibrar una celda de sedimentación gravimétrica para la medición de distribución de tamaño de micro partículas, utilizando componentes estándares. Esto con el fin de usarla en aplicaciones en la mediana y pequeña industria de los países en desarrollo. La calibración se realizó mediante el uso suspensiones de esferas de vidrio calibradas en agua y los resultados fueron comparados con los obtenidos mediante espectrometría láser, análisis de imágenes y centrifugación analítica. Estos resultados mostraron que la celda entrega resultados comparables a los de los otros métodos mas sofisticados.

Palabras clave: medición de tamaño, componentes estándares, micro partículas, calibración

\section{Construction and Calibration of a Cell to Determine Size Distribution of Micro Particles}

The development and the results obtained during the construction and calibration of a gravitational sedimentation cell to determine size distribution of microparticles, using standard components, are presented and analyzed. This with the objective of using the device in applications by the mediumsize industries of developing countries. The cell is calibrated using suspensions of glass spheres in water and the results were compared with those obtained by laser spectrometry, image analysis and analytical centrifuges. The proposed cell gave results that are comparable to the other more sophisticated methods. 


\section{INTRODUCCIÓN}

La determinación de la distribución de tamaño y del diámetro medio de partículas es un problema que está adquiriendo cada vez una mayor importancia para la industria de procesos como es la minería, la producción de celulosa y de alimentos semielaborados, ya que estas industrias emplean, entre otras, tecnologías de molienda, de separación de fase, de transporte neumático y/o hidráulico y obviamente tecnologías de descontaminación y medioambientales. La razón de esta importancia radica en el hecho que en la práctica industrial no existe en un determinado polvo con un tamaño o diámetro único de partícula, sino que existe un tamaño o diámetro que pretende representar a un conjunto de partículas de dimensiones similares, lo que implica que para la misma medida de tamaño nominal, existe un porcentaje con un tamaño mayor y otro con uno menor al valor nominal. La calidad de un determinado proceso de reducción de tamaño o de clasificación por tamaño sólo puede ser evaluado a partir de la medición de la distribución de tamaño y del diámetro medio de partículas que egresan del proceso. Este problema es singularmente más dificultoso a nivel de micro partículas debido fundamentalmente a los fenómenos físico-químicos que se presentan en partículas cuyo diámetro medio es menor a 50 $\mu \mathrm{m}$. (Bernhardt, 1990; Schubert et. al., 1990; Stiess, 2009).

Actualmente existe tecnología capaz de medir con buena precisión e incluso en tiempo real la distribución de tamaño y el diámetro medio de micro partículas, esta tecnología es elevado costo y por lo tanto no esta al alcance de las Pequeñas y Medianas Industrias (PYMes) latinoamericanas, las que de contar con equipos de medida de relativo bajo costo y adecuada precisión, podrían mejorar significativamente sus procesos, lo que redundaría en una reducción de sus requerimientos energéticos y en una mejora en la calidad de los productos, lo que incrementaría la competitividad de estas industrias a través de una reducción de sus costos de producción. (Ruscitti et. al., 2008; Steinke et. al., 2009; Blume y Brümmer, 2010). Esta es la motivación de este trabajo, en que se presenta el desarrollo de un equipo de medida de bajo costo y con una adecuada precisión para determinar de la distribución de tamaño y el diámetro medio de micro partículas, el que se calibró según la norma DIN 66111, (1989).

\section{DISEÑO CONCEPTUAL}

El equipo construido y ensayado corresponde a una celda que utiliza como método de separación de las partículas del fluido la sedimentación gravimétrica, lo que permite evaluar distribuciones de tamaño de partículas con diámetros medios mayores a $1 \mu \mathrm{m}$. A su vez, el sistema de detección de posición de las partículas y por ende su distribución de tamaño es el método de extinción fotométrica a partir del procesamiento de sucesivas imágenes obtenidas mediante una cámara Web-Cam CMOS, de una cubeta transparente donde se produce la sedimentación de las partículas. Esta cubeta es iluminada por una fuente de luz ubicada frente a la posición de la cámara, dejando entre esta fuente de luz y la cámara la cubeta que contiene la suspensión con las partículas cuyo tamaño se desea medir.

El principio de medida del equipo se basa en medir la velocidad de sedimentación del denominado espejo de separación, que corresponde a la frontera que se establece durante un proceso de sedimentación entre la suspensión que contiene material particulado y la porción de fluido que va quedando libre de partículas suspendidas. De manera que es necesario establecer la posición espacial para sucesivos intervalos de tiempos de este espejo de separación, de modo de obtener perfiles temporales de la posición de las partículas a medir y a partir de estos establecer la velocidad de sedimentación, que es dependiente del tamaño del sistema de partículas que sedimenta. (Rose, 1950; Dettmar y Lode, 1962; Staudinger y Hangl, 1986).

Conceptualmente la celda de sedimentación presenta la siguiente disposición que se indica en la Figura 1. 


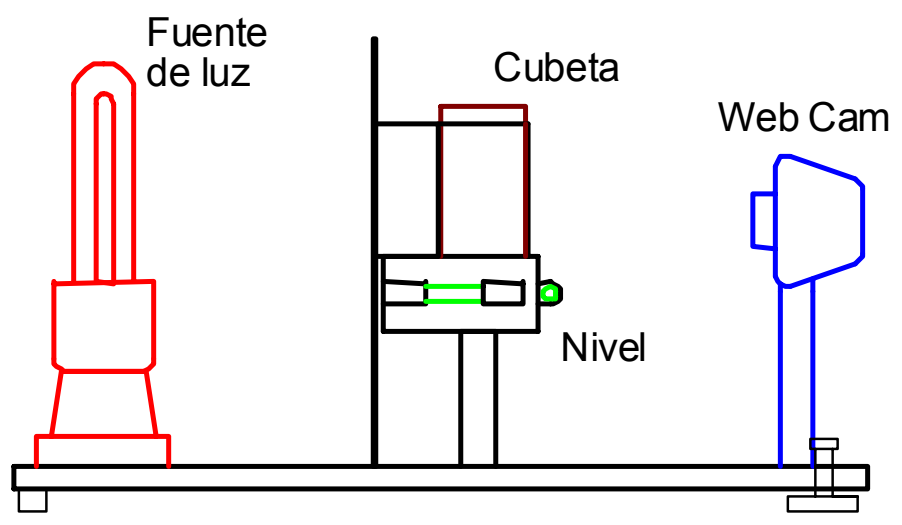

Fig. 1: Esquema conceptual de la celda de sedimentación

\section{ELEMENTOS TEORICOS}

Dado que en esta la celda de sedimentación intervienen tanto principios de la mecánica de los fluidos como de la óptica, por lo que estos serán revisados sucintamente a continuación:

El principio físico del movimiento de las partículas se basa en el principio de sedimentación ocasionada por el campo gravitatorio, el que es posible modelarlo asumiendo una partícula representativa del sistema que sea rígida, lisa, con forma esférica y que se sumerja en un fluido viscoso del tipo newtoniano. Ergo, se hace posible considerar párale balance dinámico de fuerzas, sólo los términos de cambio de cantidad de movimiento, peso de la partícula, fuerza de flotación y de arrastre viscoso. Esto da lugar a la Ec. (1), donde z representa la posición de la partícula en el sentido de la aceleración de gravedad (Bernhardt, 1990; Hoffmann y Husemann, 2003; SalinasSalas, 2005).

$$
\mathrm{m}_{\text {partícula }} \cdot \frac{\mathrm{d} \overrightarrow{\mathrm{z}}(\mathrm{t})}{\mathrm{dt}}=\overrightarrow{\mathrm{W}}_{\text {partícula }}-\overrightarrow{\mathrm{F}}_{\text {flotación }}-\overrightarrow{\mathrm{F}}_{\text {arrastre }}
$$

Como todo movimiento de un cuerpo sólido, en este caso una partícula esférica en un medio viscoso, que parte de un estado de reposo, se presenta una primera fase de movimiento, donde se inicia el movimiento, consecuentemente la velocidad de la partícula es variable con respecto del tiempo y la posición, la que es producto de una progresiva desaceleración la partícula a medida que ésta se sumerge en el fluido. Por lo que se tiende a un estado de equilibrio dinámico, donde el peso de la partícula se ve compensado por la acción de las fuerzas de flotación y de arrastre, alcanzando la partícula una velocidad de sumergimiento constante, a la que se le denomina velocidad terminal. El tiempo que dura esta etapa transiente es del orden de micro segundos para micro partículas, por lo que en términos prácticos, se puede despreciar este intervalo de tiempo para fines de una medición de densidad de tamaño de partículas (Bernhardt, 1990; Hoffmann y Husemann, 2003; Salinas-Salas, 2005).

El problema de evaluar matemáticamente la solución de la Ec. (1), fue resuelto analíticamente por Stokes en 1850, para un régimen de escurrimiento laminar del fluido por sobre la esfera con valores del número de Reynolds menores a 0,25. De manera que a partir del balance de fuerzas planteado en la Ec. (1), considerando el término de impulso como nulo, se puede obtener la velocidad de sumergimiento terminal a partir de la solución de la Ec. (1), la que se denomina como velocidad de Stokes y que toma la forma de la ecuación (2) y que tiene por dirección de movimiento el definido por el campo gravitatorio (Bernhardt, 1990; Hoffmann y Husemann, 2003, Ripperger et al., 2002; Salinas-Salas et al., 2005).

$$
v_{S t}=\frac{\left(\rho_{p}-\rho_{f}\right) \cdot x^{2} \cdot g}{18 \cdot \mu}
$$


Respecto a los elementos ópticos que deben tenerse en cuenta para el sistema de detección de la posición de las partículas y por ende de su tamaño, el fundamental corresponde a la Ley de Lambert-Beer, la que indica que existe un rango de concentración de material partículado, llamada zona de linealidad de la suspensión, donde se cumple la proporcionalidad entre el logaritmo natural de la intensidad luminosa que atraviesa la suspensión divido por la intensidad luminosa que cruza por el fluido libre de partículas y el área transversal efectivo de la partícula, la concentración volumétrica de partículas, el espesor de la suspensión que debe el haz luminoso atravesar y la denominada función del coeficiente de extinción, el que corresponde a la división entre el área transversal efectiva de la partícula y el área proyectada por ésta sobre una superficie plana perpendicular al haz luminoso (su sombra).

Matemáticamente esta relación toma la forma de la Ec. (3).

$\ln \left(\frac{I}{I_{0}}\right)=-K(x) \cdot A_{p} \cdot C_{v} \cdot L$

Cabe indicar que la función coeficiente de extinción es característica para un determinado tipo de partícula y de fluido y se puede evaluar tanto a partir de cálculos utilizando la teoría Mie, (Mie, 1908) o experimentalmente (Bernhardt, 1990; Ripperger et al., 2002; Schubert et al., 1990). Luego, el valor del tamaño o diámetro de las partículas presentes en la suspensión, se extrae a partir de la ecuación de velocidad de Stokes (Ec. (2)), al reemplazar las propiedades de las sustancias empleadas y el valor de la velocidad de sedimentación de las partículas, lo que permite despejar el diámetro de la partícula.

\section{DISEÑO Y CONSTRUCCIÓN}

La celda de sedimentación fue construida sobre una base de acero rectangular, sobre la cual se montó en uno de sus extremos una fuente emisora de luz, que en este caso una lámpara de baja energía comercial de una potencia nominal de $11 \mathrm{~W}$, a su vez, en el extremo opuesto se emplazo la cámara Web Cam CMOS, que actuara como sensor óptico que medirá el paso de luz a través de las micro partículas, lo que permitirá evaluar el cambio de la intensidad lumínica que atraviesa la suspensión con respecto al tiempo. Entre ambos se construyo un emplazamiento dotado de niveles de burbuja que alojara una cubeta rectangular, la función de los niveles es asegurar que la dirección de la sedimentación de las partículas coincida con la línea de acción del campo gravimétrico. La cubeta se construyo a partir de dos materiales, el primero es vidrio de cuarzo, el que se utilizó para las caras que son iluminada y sensada, (las de mayor área de la cubeta), mientras que su base y sus costados se construyeron en material plástico (Technyl). El mecanizado de la cubeta se realizo con la mayor precisión posible a fin de asegurar el paralelismo entre las placas de vidrio y no generar algún grado de interferencia óptica por falta de paralelismo. Como adherente y sello se empleo silicona. Los elementos eléctricos que alimentan la lámpara y el cable de señal de la cámara con su terminal USB, se dispusieron en uno de los extremos de la placa, a la que se doto de un emplazamiento de manera de facilitar el transporte de la celda de sedimentación. Respecto al ingreso de la suspensión a la cubeta, este se realiza por la parte superior de ésta, por simple vaciado desde el vaso de preparación de la muestra.

La Figura 2 muestra una fotografía de la celda de sedimentación y de la cubeta. 


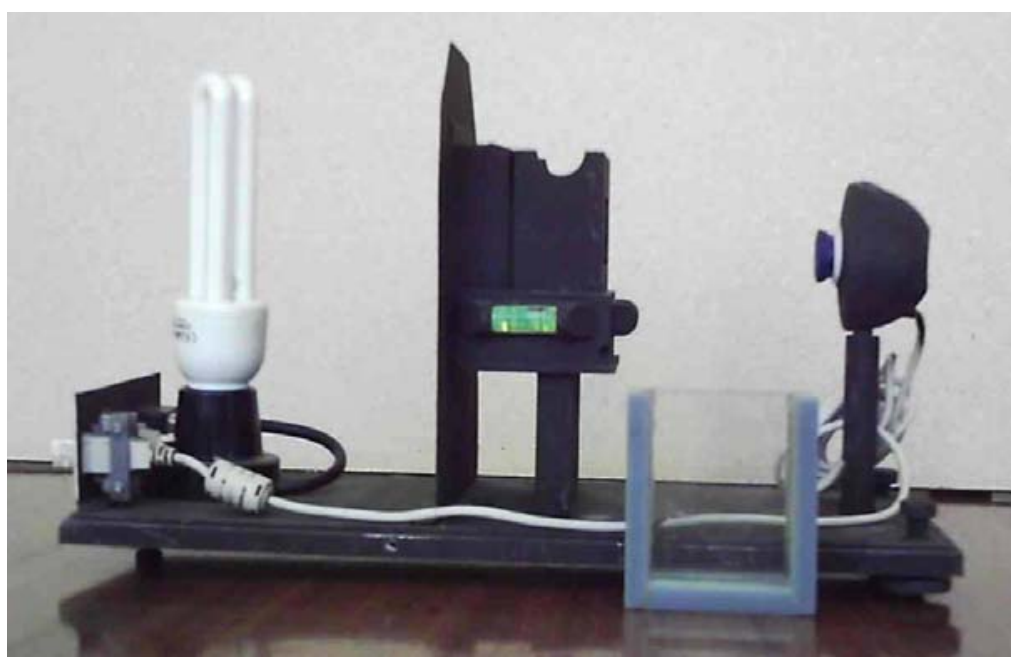

Fig. 2: Fotografía la celda de sedimentación

La adquisición de las sucesivas imágenes o fotografías en el tiempo se resuelve a través del software específico de la cámara, el que permite programar la captura de imágenes sucesivas a intervalos de tiempo prefijados y enviar estas imágenes a un computador personal, donde se efectuara el procesamiento de éstas y los cálculos matemáticos que permitirán la transformación de estas imágenes en una densidad de distribución incremental de tamaño de partícula. La Figura 3 , muestra dos imágenes sucesivas del espejo de separación que se genera durante el proceso de sedimentación y de la metodología de medición de la velocidad de éste a través del conteo de pixeles.
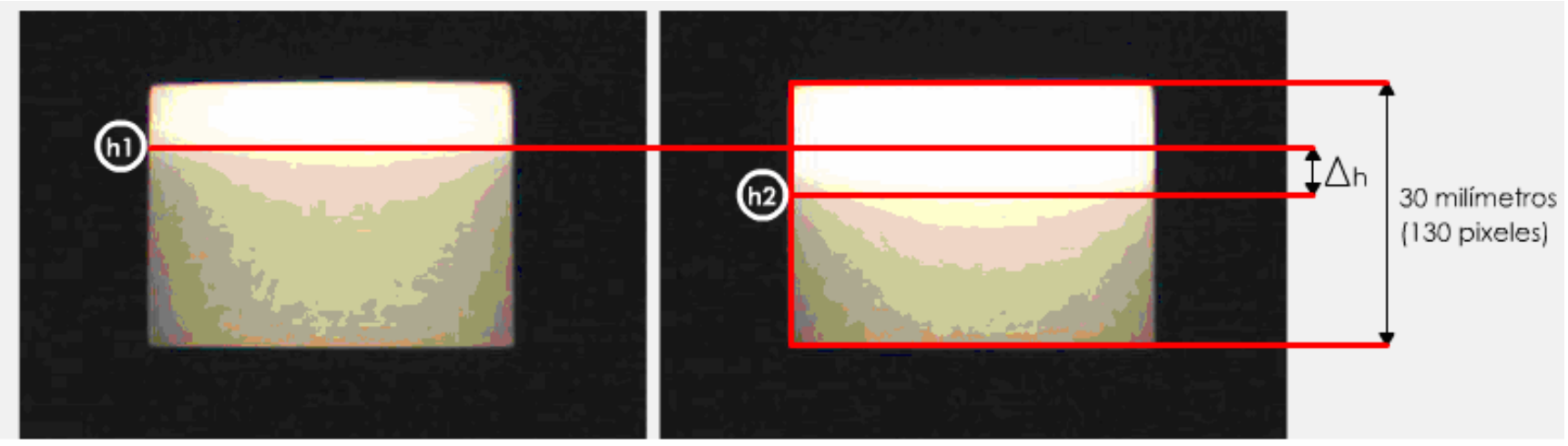

Fig. 3: Imágenes del espejo de separación, dado que la sustancia que sedimenta es vidrio, genera

Para determinación de la densidad de distribución incremental de tamaño de partícula fue necesario desarrollar un software específico de que permitiera realizar las operaciones de manejo de imágenes y calculo, el que se desarrollo en lenguaje en $\mathrm{C}++$, cuyos pasos más relevantes son los siguientes:

i) Lectura de la imagen digital y transformación a escala de grises

ii) Cálculo de la intensidad lumínica en función de la altura del espejo de separación (perfiles de intensidad)

iii) Cálculo de la distribución de velocidades de sedimentación a partir de cambio temporal de los perfiles de intensidad

iv) Cálculo de la densidad incremental de tamaño del sistema de partículas 
La primera operación implica realizar una manipulación digital de la imagen capturada, ya que la cámara utilizada entrega una señal en colores, por lo que resulto necesario por consideraciones ópticas utilizar la intensidad de los píxeles en forma de valores de una escala del color gris o escala de grises, para lo cual se debió emplear la siguiente relación de combinación para los canales de color de la cámara.

$$
I_{\text {gris }}=0,3 \cdot I_{\text {rojo }}+0,6 \cdot I_{\text {verde }}+0,1 \cdot I_{\text {azul }}
$$

La segunda operación que realiza el software es generar los perfiles de intensidad lumínica en función de la altura del espejo de separación para las distintas imágenes sucesivas. Esto se realiza al considerar que cada valor de la intensidad lumínica corresponde al valor medio de su respectiva línea de altura, a partir de esta consideración pueden construirse los perfiles de altura e intensidad para los distintos intervalos de tiempo, lo que permite generar un patrón temporal de perfiles como el mostrado en la Figura 4, donde se presentan los perfiles de intensidad para el espejo de separación en una sedimentación.

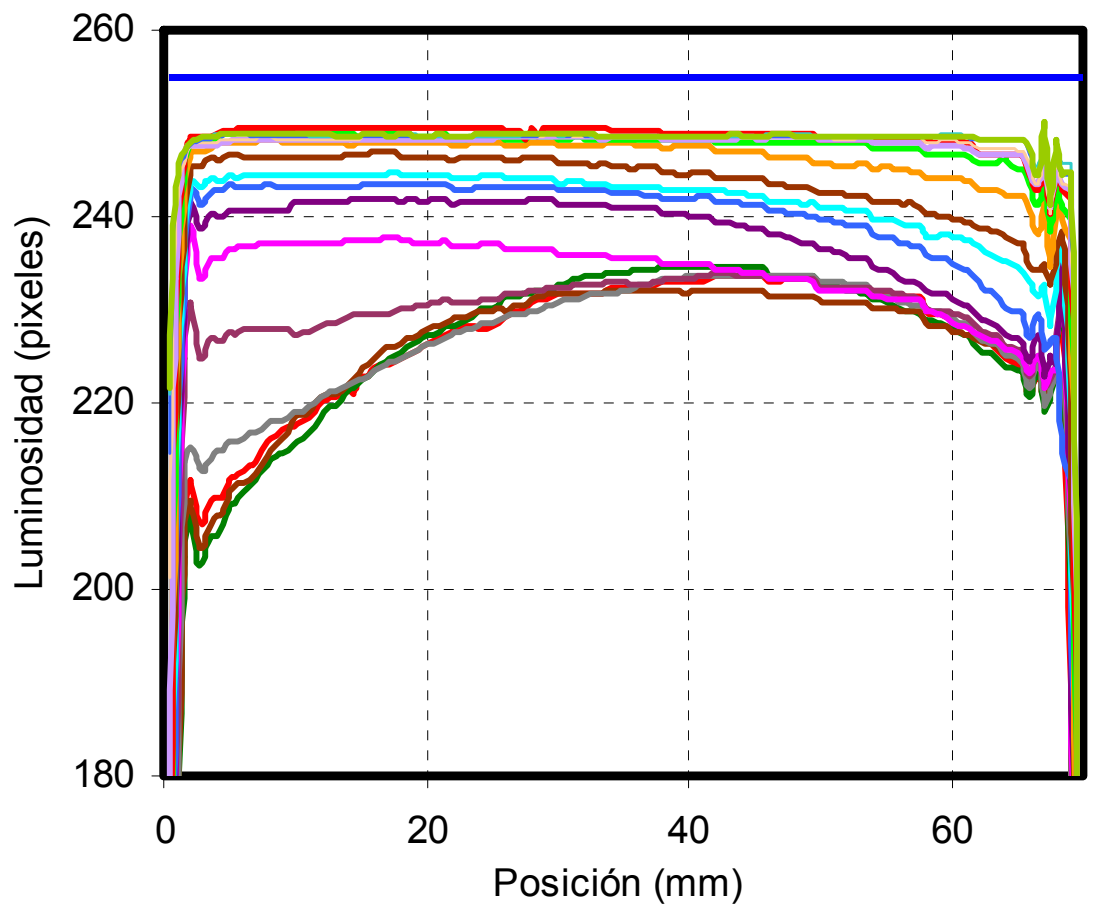

Fig. 4: Patrón de perfiles de intensidad lumínica en función del tiempo para el sistema de partículas CP 5000

Es necesario considerar que para una determinada suspensión que esta sedimentando, existen valores de intensidad lumínica en escala de grises asociados a determinadas concentraciones de partículas, de manera que los valores mínimos de intensidad en escala de grises corresponden a la zona de la suspensión donde se encuentran presentes las partículas, mientras que los valores máximos de intensidad en la escala de grises corresponden a la porción de fluido libre de partículas. Entre estos valores es posible definir un valor de intensidad lumínica que represente al espejo de separación, el que se utilizara para el cálculo de la distribución de velocidades de sedimentación.

La tercera operación correspondiente al cálculo de la distribución de velocidades de sedimentación se realiza a partir del cambio temporal de la coordenada de posición (altura) del espejo de separación.

La cuarta operación corresponde al cálculo de la densidad incremental de tamaño del sistema de partículas, lo que se realiza a partir del patrón temporal de perfiles de intensidad, ya que estos 
valores permiten determinar la distribución de la velocidad de sedimentación y a partir de ésta determinar la fracción de partículas que se encuentran en un determinado intervalo de tamaño, ya que la velocidad de sedimentación es función del tamaño de la partícula como se indica en la Ec. 2. Luego, para conocer la densidad de distribución incremental del sistema de partículas, se procede determinar la magnitud del intervalo de tamaño, a partir de las velocidades medias de sedimentación para los sucesivos intervalos de tiempo. La densidad de distribución acumulativa se determina mediante la suma de las facciones de tamaño multiplicado por el intervalo de tamaño, para luego normalizarse en una escala porcentual.

Como cualquier aparato de medida que emplee el principio de sedimentación en campo gravimétrico para la determinación de la distribución de tamaño de partículas, su uso esta limitado en la práctica para sistemas de partículas cuyos tamaños varíen entre $1 \mu \mathrm{m}$ hasta aproximadamente $100 \mu \mathrm{m}$. Para sistemas de partículas de mayor tamaño es necesario dispersarlas en un fluido que posea una viscosidad dinámica mayor a $1 \mathrm{mPa} \cdot \mathrm{s}$.

La siguiente lista muestra los principales datos técnicos de la celda de sedimentación desarrollada por el Departamento de Tecnologías Industriales de la Universidad de Talca:

Identificación del equipo

Intervalo temporal de medida

Fuente de luz

\section{Cámara Web-Cam CMOS}

Cubeta

Volumen de la prueba

Rango de concentraciones volumétricas

Rango de tamaños de partículas

Rango de densidad de partículas

Software de procesamiento de imágenes
Celda de sedimentación DTI

$10 s-600 s$

Lámpara de ahorro de energía Tipo ECO $11 \mathrm{~W}, 220$ $240 \mathrm{~V} / 50 \mathrm{~Hz}$

Creative Labs Inc., Modell PD1110, $640 \times 480$ Pixel, USB 2.0

Rectangular de vidrio de $22 \mathrm{~mm} \times 67 \mathrm{~mm}$

$70 \mathrm{ml}$

$1 \%-5 \%$

$1 \mu \mathrm{m}$ bis $100 \mu \mathrm{m}$

$2.000 \mathrm{~kg} / \mathrm{m}^{3}-10.000 \mathrm{~kg} / \mathrm{m}^{3}$

Desarrollado por el Departamento de Tecnologías Industriales de la Universidad de Talca en $\mathrm{C}++$.

\section{ENSAYOS Y CALIBRACION}

En cuanto a los materiales que se utilizaron en los ensayos de calibración, se seleccionaron a partir de la norma DIN 66111, (1989), de manera que se utilizaron partículas esféricas de vidrio soda-lima, compuesto químicamente por un $75 \%$ de oxido de silicio $\left(\mathrm{SiO}_{2}\right), 15 \%$ de oxido de sodio $\left(\mathrm{Na}_{2} \mathrm{CO}_{3}\right)$, un $9 \%$ de oxido de calcio $\left(\mathrm{CaCO}_{3}\right)$ y elementos menores, al que se denominara como CP 5000. Estas esferas de vidrio son fabricadas por Potters Industries Inc. con un diámetro medio nominal de $11 \mu \mathrm{m}$ y una densidad media del orden de $2.440 \mathrm{~kg} / \mathrm{m}^{3}$. Este sistema de partículas fue suspendido en agua tridestilada y una solución de hidróxido de potasio $(\mathrm{KOH})$, a fin de alcanzar un índice o pH 9,2, a fin de asegurar la estabilidad de la suspensión evitando con ello la formación de flóculos o cluster de partículas, durante el proceso de sedimentación que da lugar a la medición (Babick et al., 2007).

La Figura 4 muestra una fotografía obtenidas por un microscopio electrónico Zeiss Gemini 942, donde se presenta la forma y el tamaño de las partículas CP 5000 utilizadas en la calibración. 


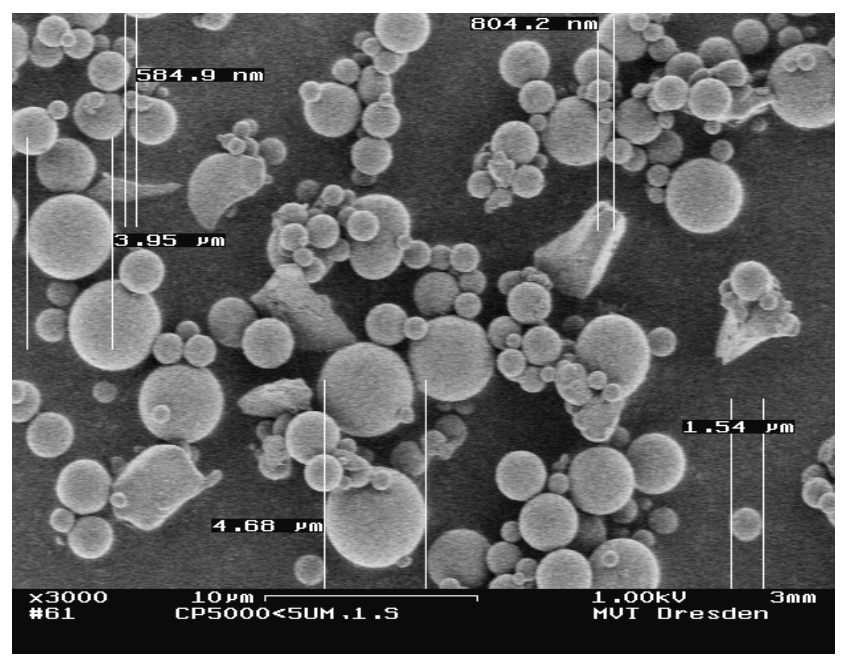

Fig. 4: Fotografía del sistema de micro partículas de CP 5000

En cuanto a la distribución de tamaño de las partículas de referencia CP 5000, esta se realizó mediante un espectrómetro Láser Helos Fa. Sympatec, cuyos resultados se muestran en la Figura 5 .

Los ensayos se realizaron en el Laboratorio de Operaciones Unitarias de la Universidad de Talca, utilizándose suspensiones de CP 5000 en agua con hidróxido de potasio como antifloculante, con concentraciones volumétricas de $0,5 \%, 1,0 \%$ y 1,5\%, utilizándose medios de dispersión mecánicos y por ultrasonido. Lo diluido de las suspensiones permite despreciar los efectos de reducción de la velocidad de sedimentación por la interacción entre partículas, lo que conduce a sumir como velocidad efectiva de sedimentación terminal a la velocidad de Stokes (Richardson y Zaki, 1954). Los resultados alcanzados tras una batería de ensayos se muestran en la Figura 5.

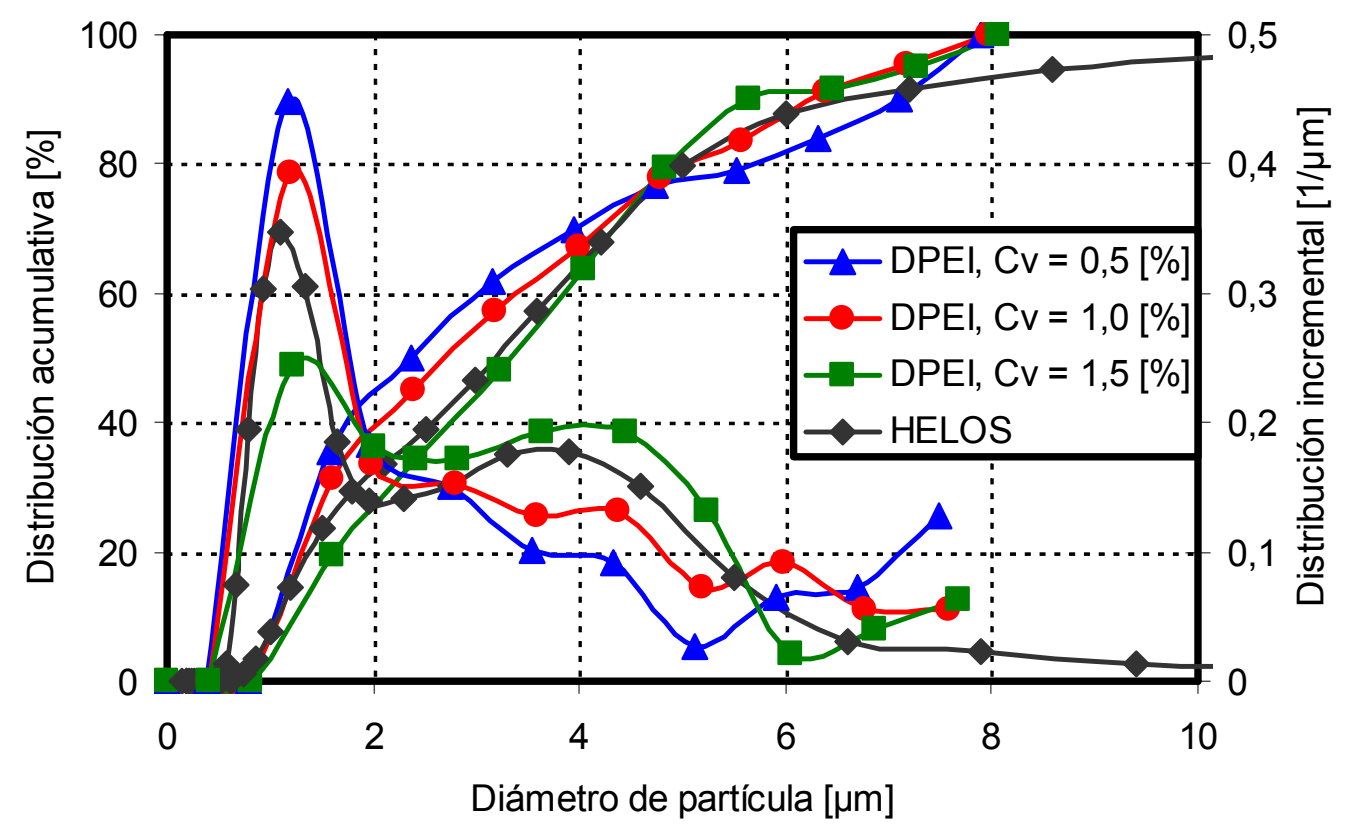

Fig. 5: Comparación de la densidad acumulativa e incremental de tamaño para el sistema de partículas de CP 5000 medidas mediante la celda de sedimentación construida y los valores de referencia

Como es posible apreciar, los resultados alcanzados por el prototipo de celda de sedimentación construida y el software desarrollado, permiten realizar una adecuada medición de la distribución de tamaño de partícula, ya que los valores absolutos de tamaño alcanzados y la tendencia de la densidades de tamaño de partícula, tanto la acumulativa como la incremental, corresponden 
adecuadamente a los valores nominales entregados por el fabricante del material de referencia, así como a los valores medidos por lo equipos de control y a los valores suministrados por los métodos de referencia que se utilizaron durante el desarrollo de los ensayos. De hecho aparece claramente la bimodalidad del material de calibración, en cuanto a los valores de punta de los modos el error cometido no supera el $5 \%$, lo que se puede considerar como adecuado considerando el tipo de celda desarrollada. Cabe indicar que los valores de tamaño de partícula entregados por la celda de sedimentación tienen una tendencia a ser mayores que la del material de referencia, esto puede deberse a que la dispersión del material particulado CP 5000, no fue perfectamente duplicada respecto de la que se realizó cuando se referenció el material en el Laboratorio de Partículas del Grupo de Trabajo de Técnicas de Procedimientos Mecánicos de la Universidad Técnica de Dresde (Arbeitgruppe für Mechanische Verfahrenstechnik de la Technische Universität Dresden).

\section{CONCLUSIONES}

De los resultados alcanzados en el prototipo construido se pudo concluir que su aplicación para la evaluación de la distribución de tamaño de micro partículas fue no solo eficiente, sino que preciso. Además, presentó las ventajas de su simplicidad de construcción, bajo costo, relativa facilidad de operación y con niveles de precisión satisfactorios, ya que el error medio cometido con el sistema de partículas CP 5000, reprodujo su modalidad y con un error medio menor a un $5 \%$.

En cuanto a las desventajas del equipo, éstas radican en la larga duración de cada ensayo, lo que incrementa las posibilidades que éste sufra alteraciones externas, tales como la formación de corrientes convectivas por efecto de calor que ingrese a la suspensión o simplemente el efecto de vibraciones mecánicas sobre la celda.

Cabe indicar que al realizarse los experimentos de acuerdo a la norma DIN 66111 (1989), el utilizar un material de referencia calibrado y equipos de control externos como el microscopio electrónico y el espectrómetro láser, permite tener confianza en los valores alcanzados, ya que estos mostraron un muy buen nivel reproducibilidad.

\section{NOMENCLATURA}

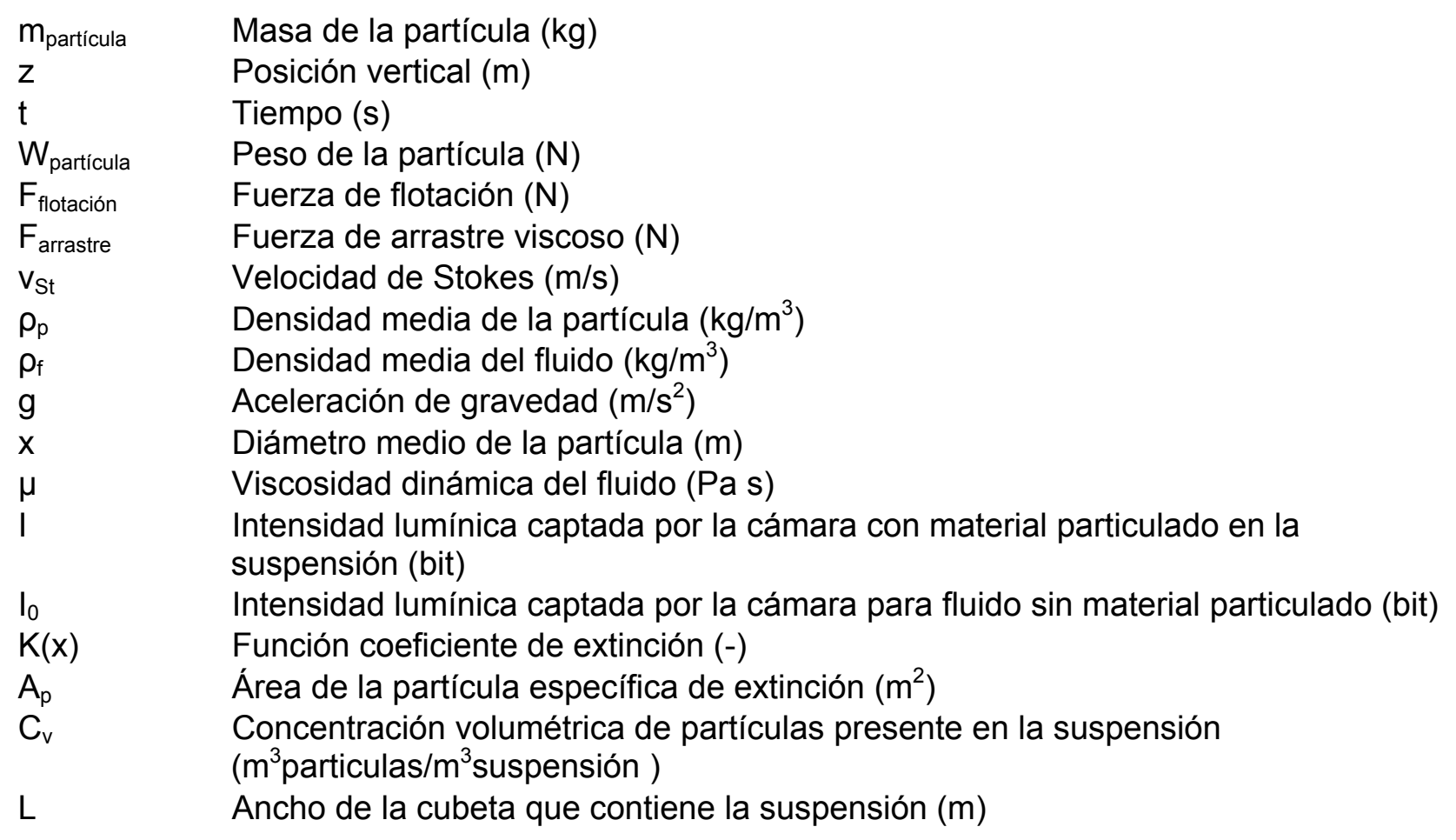




\section{AGRADECIMIENTOS}

Los autores expresan sus agradecimientos al Grupo de Trabajo de Técnicas de Procedimientos Mecánicos de la Universidad Técnica de Dresde, (Arbeitgruppe für Mechanische Verfahrenstechnik an der Technische Universität Dresden), por las facilidades prestadas para el desarrollo del trabajo experimental.

\section{REFERENCIAS}

Babick F., Salinas Salas G., Sobisch T., Lerche D., Particle interactions in dispersions of micro and nanoparticles, PARTEC 2007 - Word Congress on Particle Technology, Nürnberg, (2007)

Bernhardt C., Granulometrie, Deutscher Verlag für Grundstoffindustrie, (1990)

Blume H., Brümmer R., Lehrbuch der Bodenkunde. Reaktive Oberflächen und Oberflächenbehandlung, Spektrum Akademischer Verlag, Heidelberg, (2010)

Norma DIN 66111 sobre Métodos de Sedimentación, DIN Norme 66111 Über Sedimentationsmethoden, Alemania, (1989)

Dettmar, H.-K.; Lode, W., Lichtstreuung und Teichengrössenverteilung kugelförmiger Teilchen, KolloidZ. und Z. f. Polymere 188, pp. 28, (1962)

Hoffmann B., Husemann K., Sinkgeschwindigkeit kugelförmiger Teilchen in Suspension bei laminar bzw. turbulenter Umströmung - Exakte Lösung der Bewegungsgleichung , Chem. Ing. Techn. 75, 3, pp. 757-763, (2003)

Mie, G., Beiträge zur Optik trüber Medien, speziell kolloidaler Metallösungen, Ann. D. Physik 25, 3, pp. 377 - 445, (1908)

Richardson, J. F., Zaki, W. N., Sedimentation and Fluidisation. Part I, Trans. Inst. Chem. Eng., pp. 3553, (1954)

Ripperger S., Salinas-Salas G., Stintz M., Wessely B., Untersuchung der Sedimentation von nanoskaligen Partikeln Zentrifugalfeld, F \& S (Filtrieren und Separieren) 16, 5, pp. 230-236, (2002)

Rose, H. E., The design of photo-extinction sedimentometers, Engineering 169 (1950)

Salinas-Salas G., Stintz M., Wessely B., Aplicación del método fotométrico para la detección de la distribución de tamaño de micro partículas, Información Tecnológica, Vol. 16 N²4, pp. 59-68, (2005)

Salinas-Salas G., Babick F., Espinosa F., Stintz M., Determinación del exponente de la función de obstrucción para partículas sub-micrométricas no coloidales, Información Tecnológica, Vol. 20 №2, pp. 19-27, (2009)

Schubert H., Heidenreich E., Liepe F., Neeße T., Mechanische Verfahrenstechnik, Deutscher Verlag für Grundstoffindustrie, (1990)

Staudinger, G.; Hangl, M.: Quick optical measurement of the sedimentation characteristics of particles. Proceed. $1^{\circ}$ World Congress on Particle Technology, Nürnberg, (1986)

Steinke L., Wessely B., Ripperger S., Optische Extinktionsmessverfahren zur Inline-Kontrolle disperser Stoffsysteme. Chem. Ing. Tech. 81 pp. 735-747, (2009)

Stiess, M.: Mechanische Verfahrenstechnik-Partikeltechnologie. Springer-Verlag, Berlin, Heidelberg, (2009)

Ruscitti O., Franke R., Hahn H., Babick F., Richter T., Stintz M., Zum Einsatz der Partikelmesstechnik in der Prozessintensivierung, Chem. Ing. Tech. 80, 1-2, pp. 191-199, (2008) 